Indonesian Journal of Electrical Engineering and Computer Science

Vol. 11, No. 3, September 2018, pp. 839 847

ISSN: 2502-4752, DOI: 10.11591/ijeecs.v11.i3.pp839-847

\title{
A Radio Signal Strength Based Localization Error Optimization Technique for Wireless Sensor Network
}

\author{
Sudha H. Thimmaiah ${ }^{1}$, Mahade van $\mathbf{G}^{2}$ \\ ${ }^{1}$ Department of Telecommunication \& Engg., Dr. AIT, VTU, Bangalore, India \\ ${ }^{2}$ Annai college of Engg., Kumbakonam, India
}

\begin{tabular}{|c|c|}
\hline Article Info & ABSTRACT \\
\hline & \multirow{8}{*}{$\begin{array}{l}\text { Wireless Sensor Networks (WSN) is useful in collecting data from various } \\
\text { sensor devices that are distributed over a network which is generally } \\
\text { positioned in a stationary manner. Wireless sensor based communication } \\
\text { system is an ever growing sector in the industry of communication. Wireless } \\
\text { infrastructure is a network that enables correspondence between various } \\
\text { devices associated through an infrastructure protocol. Finding the position or } \\
\text { location of sensor node (Localization) is an important factor in sensor } \\
\text { network for proving efficient service to end user. The existing technique } \\
\text { proposed so for adopt AOA (Angle of Arrival), TOA (Time of Arrival) etc, } \\
\text { suffers in estimating the likelihood of localization error and induces high cost } \\
\text { of deployment. To cater this in this work the author proposes a cost effective } \\
\text { RSS (Received signal strength) based localization technique and also } \\
\text { proposes an adaptive information estimation to reduce or approximate the } \\
\text { localization error in wireless sensor network. The author compares our } \\
\text { proposed localization model with existing protocol and analyse its efficiency. }\end{array}$} \\
\hline Received May 9, 2017 & \\
\hline Revised May 26, 2018 & \\
\hline Accepted Jun 11, 2018 & \\
\hline Keywords: & \\
\hline Localization & \\
\hline Radio channel measurement & \\
\hline WSN & \\
\hline
\end{tabular}

Copyright (C) 2018 Institute of Advanced Engineering and Science. All rights reserved.

Corresponding Author:

Sudha H. Thimmaiah,

Department of Telecommunication \& Engg., Dr. AIT, VTU,

Bangalore, India.

Email: sudhacseait@rediffmail.com

\section{INTRODUCTION}

A WSN comprise of thousands or a more sensory devices and a little number of information gathering gadgets or nodes [21]. The sensor devices have the type of low-power, cost, and size and are intended to do a scope or provide various sensory application services such as surveillance system for military and police services, monitoring environmental application service, monitoring or counting number of habitat in forest, used in fire safety alarm and it is used to detect fire, etc. This has been enabled by the availability, especially in recent years, of sensors that are small in size. These nodes are furnished with wireless interface (WI) that enables them to connect through each other to form a wireless infrastructure. The sensor devices accumulate the data of interest locally and after that forward the sensory data collected over a remote medium to a remote information gathering nodes (base stations or sinks), where data is analyzed with a specific end goal to decide the universal status of the area that have been sensed.

In order to develop an efficient QoS based application for WSN the following issues and challenges needs to be addressed [22]. Communication among sensor devices i.e. huge amount packet transmission in wireless sensor network is merely not practical contrasting to other wireless infrastructure devices such as router, network interface card, etc., since these sensor devices are designed to cater low power requirement. Networking i.e. sensor networks often lead to volatile outcome due to topology dynamic as result it leads to an unbalance consumption of energy, higher collision rates, and channel contention among nodes. Data confidentiality i.e. To safeguard the obtained data information from unauthorized user. To achieve this various userauthentication and cryptographic protocol is been proposed for sensor network. Management i.e. sensor network require proficient protocol strategy to extract their potential of following thing such power 
management, radio device interference management, battery management of sensor devices, hardware and software bug management, node failure, data loss due node failure etc...

There are challenges in wireless sensor network that need to be consider in localization of sensor nodes such as reduce the error in approximating distance i.e. the path loss model can be determined, where the prototype parameters can be evaluated based on obtained data by using measurement of RSS [8], [9] and [12], TDOA/TOA [19], [20] and AOA [17], [18]. Such prototype fixes the distance from the unknown node to at least few nodes with known locations. Then, classical methodologies may be employed to find the position of the unknown device. The drawbacks of this kind of model are low scalability, topology sensitivity and unreliability, [12], [13]. Consider and accepts the noisy measurement but to reduce the error in estimating the location they perform a global optimization [9]-[11], [17] and [18]. One well known methodology is based on MDS (multidimensional scaling) [10], [13], [15]. These methodology aids at finding a low-dimensional location mapping from a high-dimensional distance measurement space by reducing the pairwise detachment errors [14]. These distances matrix evaluated form a matrix that could be used to restructure a 2-D or 3-D coordinate infrastructure by multidimensional scaling. In this scenario the processes such as reflection, shifting or the combination of both can be used to keep the remoteness among the points invariable.

WSNs guarantee to enhance the correspondence of critical data in various industries of uses. As of late, WSN-based SOA (service oriented based architecture) has ended up progressively key for building adaptable and effective application administrations [1] where application administrations live in distinctive layers of data frameworks [3]. Consistently incorporating application administrations into WSNs is an interesting issue of administration based applications. Numerous SOA-based applications, for example, information storage and management have been explored in [3] and [4]. In [5], a SOA is proposed for industrial purpose, where WSNs are connected as an application administrator. SOA based applications such as Monitoring ecological application, GPS based application, also, medicinal services have been considered services in [5]-[7].

In sensor network the localization of node has turned into a crucial prerequisite for providing effective service to end user. Many location based service are provided to localize the node but it is practically difficult to provide an effective localization technique. Recently many localization techniques have been proposed based on GPS (global positioning system) [10], [11] to solve the localization problem as in [8]-[10] but these technique suffers from energy efficiency issues and the computational cost is also high and it is very expensive to localize the node due topology dynamic as follows (large number of nodes, equipping the sensor with GPS, etc.). An alternative approach based on distance measurement of reference and unknown node is performed by the following technique TDoA (Time difference on arrival), ToA (time on arrival), RSS (Received signal strength) and so on.

The localization technique based on TOA framework [19], [20] needs supplementary devices or hardware to ensure synchronization among a transmitting and receiving device. More essentially, a tiny mistake in estimating time will cause a substantial or noteworthy ranging error. Additionally, the localization technique based on TDOA offer comparable downsides with TOA frameworks, on the grounds that they all include huge cost for equipment. Also, TDOA/TOA techniques for the most part require a highly dense deployment of sensing devices, because of their shorter signal propagation distances. i.e. the propagation distance of ultrasound is roughly 15-25 feet. Besides, AOA [17], [18] frameworks evaluate the distance between nodes by denoting to the relative points measured through an edge measuring node. In spite of the fact that AOA frameworks can accomplish high localization accurateness, they are not suitable for vast scaled or exceptionally dense sensor networks because of their complicated design and induce high cost or expenses. The localization techniques based on RSS, on the other hand, overcomes or conquers these inadequacies or shortcomings that are mentioned above. The principle of the RSS methods is to utilize some RF (Radio Frequency) signal propagation framework derived from either hypothetical derivation or theoretical inference or empirical or exact results to make a an interpretation of RSS to quantify the remoteness without requiring additional ranging hardware. Such systems are cost-effective and can be helpfully executed since most RF IC-chips used in WSN devices can directly deliver the data info of RSS.

In this work the author propose a cost effective RSS based localization technique and also proposes an adaptive information estimation to reduce or approximate the localization error in wireless sensor network.

The paper organization is as follows: The literature survey is presented in section two. The proposed localization models are presented in section three. The results and the experimental study are presented in the section four. The concluding remark is discussed in the last section. 


\section{RELATED WORK}

There are several approaches have been proposed to approximate localization error for WSN based application service in recent times in order to reduce localization error and provide QoS to its end user which are surveyed below.

In [22] they presented a localization model for figuring out the location of sensor devices in sensor network. There model adopt a trust based reply messages sent by the adjacent devices that are either a localized device or anchors node against the message invitation sent by the device that aiming or considering to localize itself. They adopted a distributed, asynchronous localization methodology hence the topology information parameter is not necessary. They performed better than some of the shortest path algorithm that have been presented for localization such as CIL [23] but it causes a node localization failure if an neighbor anchor nodes fails and it also suffer from high computational complexity for calculating the localization confidence parameter.

In [44] author want to reduced the cost in WSN by avoiding the use of GPS for that author propose two algorithm named as bio-inspired optimization algorithm in WSN which is helpful in node localization, and other is frog leaping algorithm. This algorithm is easy to be used in multidimensional search in WSN. Received signal strength indicator (RSSI) proposed in [45] which is based on that constraint particle swarm optimization, here they used similar method to calculate the coordinate value, in this method distance of anchor point is measured and difference is calculated based on actual value and measured value and then after obtained the coordinate value of unknown nodes.

A few localization technique based on hybrid methodology strategy are also considered [24]. These strategies provide support for both the range free based and range based methodologies. In [26] an anchor free localization strategy is developed in this strategy the localization is done based on node connectivity, direction also consider distance among neighborhood devices and does not required and added hardware for node localization. This methodology still suffers in term of execution time needed for the calculation or approximating the error. Due to this many artificial intelligence based localization techniques that has been proposed such as [25] GA (genetic algorithm), [27] PSO (Practical swarm optimization), [28] ANN (artificial neural network), etc. though these technique offer better localization optimization but they are generally designed as a centralized approach and it suffer from NP-Hard problem and obstacle tracking and anchor node identification.

The Range-free localization technique estimate the node info among normal devices by obtaining through connectivity with the adjacent devices which does not need any added infrastructure or tools thus reducing cost and consumption of energy. For example the convex position estimation (CPE), the area based approach (centroid DV based positioning (DV hop), and Monte Carlo localization (MCL) [29], [30], [31] etc.... these algorithms reduce the cost and network overhead but in order to achieve good positioning accuracy, it needs to decorate the large number of anchor nodes to overcome these accuracy issue in localizing node range based method is adopted or considered over range free based localization.

The range based technique requires an added infrastructure or hardware but by considering that it can offer high precision or accuracy than range free based methodologies. Considering this there are various equipment or devices available such as, they exploit the AoA, the ToA, the TDoA and the RSS. A review and evaluation of various methodologies is presented in [32-37].

The AoA is a method for defining the radio frequency wave direction of propagation. The state of art methodology to measuring angles is by rotating on their axis which is alike to a radar station, to consider directional antennas. Another way is by deciding radio frequency wave flow direction of propagation on an antenna array. This methodology can't be utilized as part of infrastructure formed by tiny devices with just a solitary antenna setting.

The ToA and TDoA methodology exploit the association (link) among the transmission time and distance when the speed of propagation is acknowledged. If the sender and receiver know the time when the transmission starts, the ToA propagation can be used to compute the distance. ToA schemes consider ultrasound which uses signals that move at a slower speed. The source devices send a signal to the destination devices and, in return, the destination devices send a signal back to the source devices. Next, the inter devices distance is computed based on [35]. To provide high precision and accuracy synchronized clocks is needed at both source and destination device. To overcome these TDoA approach is considered which uses an implicit synchronization technique by directly giving the initiation of transmission information to the destination devices. This is practical if and only if two transmission devices use different propagation speed and can work only in densely deployment area.

A combination of TDoA and AoA Methodologies is developed [39] to enhance the accuracy of localization of sensor devices. It ought to be noted that both the TDoA and ToA are too difficult to be realistic in wireless sensor infrastructure with constrained computational and communication capabilities. The other approach developed in [38] is a system that consolidates the Time Frequency Difference of Arrival 
(TFDA) and the linear Frequency Modulation Continuous Wave (FMCW) procedures. In this methodology a distance difference from one device to another is computed, rather than a computing of an inter-device distance.

In [40] DV-Hop localization scheme which consider between beacon nodes as the mean distance per hop beacon nodes unknown node to the mean distance per jumping, jumping distance and jumping through each multiplied by the number to represent the unknown nodes and beacon nodes the distance between. In [41] they identified the drawback of [40] and proposed a new algorithm based on prioritized threshold based anchor node localization this technique improve the accuracy but suffer in estimating the ranging error. To solve the ranging error in [42], [43] they proposed a RSS based weighted centroid algorithm. There experiment result shows that RSS solves the ranging error among unknown devices for DV-Hop algorithm.

Form survey it is noted that the most common approach taking into account a standard element found in many wireless sensor nodes is the RSS. The benefit of this system is low deployment cost (no added infrastructure required), simple design, configuration and deployment.

\section{PURPOSED SYSTEM}

Here we specialize for senor node location estimation approximation by adopting RSS based pairwise measurements in a WSN. Precisely, consider a WSN of $p$ reference nodes (These nodes have a priori information about their coordinates) and $q$ blindfolded sensor nodes (These nodes does not have a priori information). Let $\alpha=S_{1}, \ldots . S_{p+q}$ be the node parameter where for a two-dimensional infrastructure it can represented as, $S_{x}=\left[i_{x}+j_{x}\right]^{B}$. Let $\varphi=\left[\varphi_{i}, \varphi_{j}\right]$ be the blindfolded device co-ordinates relative location problem estimation.

$$
\varphi_{i}=\left[i_{1}, \ldots . i_{q}\right] \quad \varphi_{j}=\left[j_{1}, \ldots . j_{q}\right]
$$

given the known reference coordinates $\left[i_{q+1}, \ldots, i_{q+p}, j_{q+1}, \ldots, j_{q+p}\right]$

In RSS based the measured acknowledged power at node $x$ communicated by node $y$ is represented as $I_{x, y}=M_{x, y}$ (milliwatts). The authors consider only subset of $Z(t)$ of nodes that create pair-wise estimations with node $t,\left(\left(B_{x, y}\right)\right)_{x, y}$ and $\left(\left(P_{i, j}\right)\right)_{i, j}$ are reserved to be upper triangular matrices, and these estimations are presumed to be statistically autonomous. Let us also consider that $B_{x, y}$ is Gaussian distributed with variance $\sigma_{B}^{2}$ and mean $n_{x, y} / m$, which is represented as

$$
B_{x, y} \sim D\left(n_{x, y} / m, \sigma_{B}^{2}\right), n_{x, y}=n\left(S_{x}, S_{y}\right)=\left\|S_{x}-S_{y}\right\|^{1 / 2}
$$

where $\sigma_{T}^{2}$ is not a function of $n_{x, y}$ and $m$ is the propagation speed, and then the author consider that $M_{x, y}$ is log-normal therefore the random variable $M_{x, y}(\mathrm{dBm})=10 \log _{10} M_{x, y}$ is Gaussian

$$
\begin{aligned}
& M_{x, y}(d B m) \sim D\left(\bar{M}_{x, y}(d B m), \sigma_{d B}^{2}\right) \\
& \bar{M}_{x, y}(d B m) \sim M_{0}(d B m)-10 e_{p} \log _{10}\left(n_{x, y} / n_{0}\right)
\end{aligned}
$$

Where $M_{0}(d B M)$ is the received power in decibel milliwatts at a reference distance $n_{0}$, generally $n_{0}=1 m$, and $M_{0}$ is calculated from the free space path loss formula [16]. $\sigma_{d B}^{2}$ is the variance of shadowing, $\bar{M}_{x, y}(\mathrm{dBm})$ is the mean power in decibel milli watts, and The path loss exponent $e_{p}$ is a function of the environment. For particular environments, $e_{p}$ can be estimated and known from prior measurements. Even though the author derive the Adaptive information estimation strategy assuming $e_{p}$ is known, it can be handled as an unknown parameter. Given (3), the load of $M_{x, y}$ is

$$
\begin{aligned}
& l_{M \mid \alpha}\left(M_{x, y} \mid \alpha\right)=\frac{10 / \log 10}{\sqrt{2 \pi} \sigma_{d B}^{2}} \frac{1}{M_{x, y}} \exp \left[-\frac{u}{8}\left(\log \frac{n_{x, y}^{2}}{n_{x, y}^{2}}\right)^{2}\right] \\
& u=\left(\frac{10 e_{p}}{\sigma_{d B} \log 10}\right)^{2}
\end{aligned}
$$




$$
\widetilde{n_{x, y}}=n_{0}\left(\frac{M_{0}}{M_{x, y}}\right)^{1 / e_{p}}
$$

Here $\widetilde{n_{x, y}}$ is the Average likelihood error of range $n_{x, y}$ given received power $M_{x, y}$.

Neither $M_{x, y}$ nor $B_{x, y}$ are presumed to be stochastic process or ergodic arbitrary variables; in fact, shadowing errors due to obstacles in the measured environment usually does not change over a period of time. The AIES gives a lower bound on the ensemble variance over different arbitrary shadowing environments. If networks with the similar relative node coordinates are implemented in many different areas, the variance of any unbiased coordinate estimator will be lower bounded by the proposed AIES.

\subsection{Adaptive Information Estimation Strategy (AIES)}

In sensor infrastructure in order to solve the problem of self-calibration, parameters of all nodes in a infrastructure must be determined. Data info comes from measurements made among pairs of nodes and also from a subset of nodes that know a priori their bounds. A infrastructure self-calibration estimator calculates the unknown nodes bounds. Let consider, distributed clock synchronization in a infrastructure could be accomplished by nodes perceiving pair-wise timing offsets when just a small number of devices are synchronous.

Precisely, consider a vector of node bounds $\alpha=\left[\alpha_{1} \ldots \ldots \alpha_{q+p}\right]$. every node has one bound. Nodes $1 \cdots q$ are blindfolded nodes, and nodes $q+1 \cdots q+p$ are reference nodes. The unidentified bound vector is $\varphi=\left[\alpha_{1} \ldots \ldots \alpha_{q}\right]$, where $\varphi_{x}=\alpha_{x}$ for $x=1 \cdots q$, Note that $\left\{\alpha_{x}, x=q+1 \cdots q+p\right\}$ is acknowledged. Nodes $x$ and $y$ make pair-wise observations $I_{x, y}$ with load $l_{I \mid \alpha}\left(I_{x, y} \mid \alpha_{x}, \alpha_{y}\right)$. The author permit for the circumstance when nodes make unfinished observations since two nodes may have limited link capacity or may be out of communication range. Let $Z(x)=\{y$ : node $y$ makes pair-wise observations with node $x$ \}.

The pdf joint conditional is defined as

$$
\begin{aligned}
& f(I \mid \alpha)=\sum_{x=1}^{p+q} \sum_{y \in Z(x) y<x} f_{x, y} \\
& f_{x, y}=\log f_{I \mid \alpha}\left(I_{x, y} \mid \alpha_{x}, \alpha_{y}\right)
\end{aligned}
$$

The adaptive strategy to obtain covariance matrix for any unbiased estimator $\varphi^{\wedge}$ is $\operatorname{cov}\left(\varphi^{\wedge}\right) \geq \mathrm{L} \_\varphi^{\wedge}(-1)$ where the adaptive information matrix is estimated as;

$$
L_{\varphi}=-J \nabla_{\varphi}\left(\nabla_{\varphi} f(I \mid \alpha)\right) B=\left[\begin{array}{llc}
l_{1,1} & \cdots & l_{1, q} \\
\vdots & \ddots & \vdots \\
l_{q, 1} & \cdots & l_{q, q}
\end{array}\right]
$$

The diagonal elements $l_{t, t}$ of $L_{\varphi}$ reduce to a solitary sum over $Z(x)$ since there are pass $\{Z(t)\}$ term in (5) that depend on $\varphi_{t}=\alpha_{t}$. The off-diagonal elements when $t=f$ as

$$
l_{t, f}=\left\{-\sum y \in Z(t) J\left[\begin{array}{c}
\partial^{2} \\
\partial \varphi_{t}^{2}
\end{array} f_{t, y}\right]\right.
$$

The off-diagonal elements when $t \neq f$ as

$$
l_{t, f}=\left\{-A_{z(t)}(l) J\left[\begin{array}{c}
\partial^{2} \\
\partial \varphi_{t} \partial \varphi_{f}
\end{array} f_{t, f}\right],\right.
$$

Where the indicator functions $A_{z(t)}(f)$, is 1 where $f=\in Z(t)$ or else it is 0 .

\section{RESULTS AND ANALYSIS}

In this section we discuss the experimental study for wireless sensor localization by using proposed localization approach. Simulation study is carried out using windows 7 enterprises 64-bit operating system with 4GB RAM and MATLAB tool is used. For the simulation study we have considered various localization parameters i.e. sensor nodes deployment area, number of nodes to be deployed, number of reference devices and localization iterations or localization attempts, Received signal strength method is considered for the measurement of localization error performance. 
To show the efficiency of the system we have conducted simulation study using varied simulation parameters. In the initial case by keeping constant the number of references devices (4) and number of nodes (4), varied the localization attempts. Figure 1 shows the localization performance of this model in terms of root mean square error. Average error of localization is given in the Figure 2. The performance of proposed localization error is compared with the existing standard localization algorithm. Table 1. shows simulation parameters Average localization performance is increased by $89.16 \%$ which can be computed by Table 2.

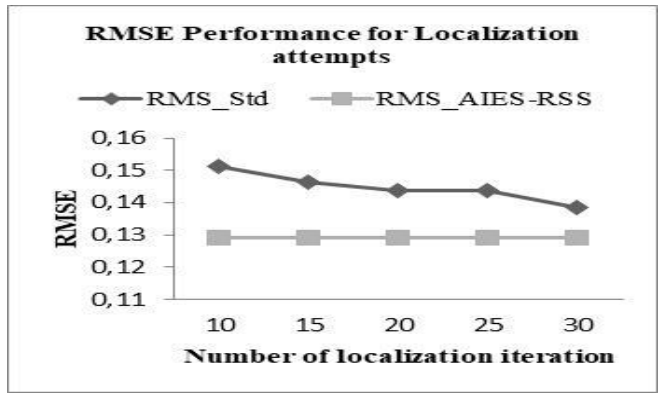

Figure. 1. Varied localization attempts

Table 1. Simulation Parameters

\begin{tabular}{lc}
\hline \multicolumn{1}{c}{ Parameter Name } & $\begin{array}{c}\text { Parameter Value } \\
\text { Considered }\end{array}$ \\
\hline Area & {$[1 \times 1]$ meters } \\
Sensors per side & $4,6,8,10$ \\
$\begin{array}{l}\text { Reference Devices } \\
\text { Localization Iteration }\end{array}$ & 4 \\
$\begin{array}{l}\text { Localization measurement } \\
\text { method }\end{array}$ & $10,15,20,25,30$ \\
\hline
\end{tabular}

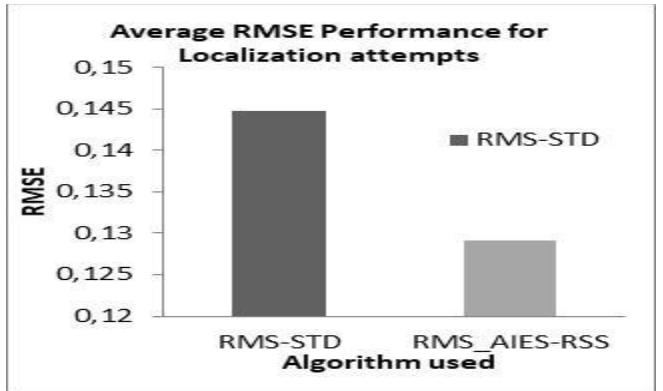

Figure 2. Average localization error

Table 2. Average RMSE Performance

\begin{tabular}{cc}
\hline Algorithm Used & Average RMSE \\
\hline RMS-STD & 0.14478 \\
RMS-AIES-RSS (proposed) & 0.1291 \\
\hline
\end{tabular}

Localization error is computed in terms of RMSE as:

$$
R M S E=\sqrt{\frac{\sum_{i=1}^{n}\left(\text { Loc }_{\text {real }}^{i} \text { Loc }_{\text {eSt }}^{i}\right)^{2}}{n}}
$$

In the 2nd scenario we have varied the number of devices or nodes by keeping the reference devices and localization attempts constant. This performance and average performance of this scenario is given in Figure 3 and 4 and average localization error is reduced by 96.30. Performance evaluation is mentioned in Table 3. Table 4 shows performance evaluation for 3rd case. Graphical representation of this case is depicted in Figure 5.

In above given Figure 5 shows the RMSE performance for varied number of devices and iterations, $\mathrm{x}$-axis shows the number of device and corresponding number of iterations. Average performance achieved is shown in Figure 6 and achieved data is given in Table 5.

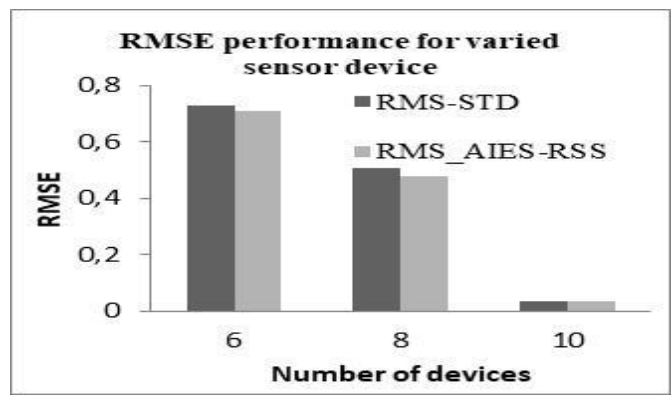

Figure 3. Varied sens or devices

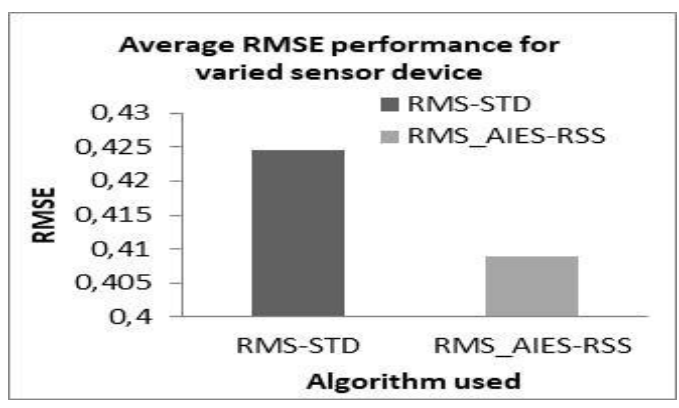

Figure 4. Average RMSE for varied sensor devices 


\begin{tabular}{cc}
\multicolumn{2}{c}{ Table 3. Average RMSE Performance } \\
\hline Algorithm Used & Average RMSE \\
\hline RMS-STD & 0.424566667 \\
RMS-AIES-RSS (proposed) & 0.408866667 \\
\hline
\end{tabular}

Table 4. Performance Evaluation for 3rd Case

\begin{tabular}{ccccc}
\hline $\begin{array}{c}\text { Number } \\
\text { of } \\
\text { devices }\end{array}$ & $\begin{array}{c}\text { Reference } \\
\text { devices }\end{array}$ & $\begin{array}{c}\text { Localization } \\
\text { attempts }\end{array}$ & $\begin{array}{c}\text { RMS- } \\
\text { STD }\end{array}$ & $\begin{array}{c}\text { RMS- } \\
\text { AIES- } \\
\text { RSS }\end{array}$ \\
\hline 6 & 4 & 20 & 0.0796 & 0.0712 \\
8 & 4 & 25 & 0.0475 & 0.0479 \\
10 & 4 & 30 & 0.0387 & 0.0356 \\
\hline
\end{tabular}

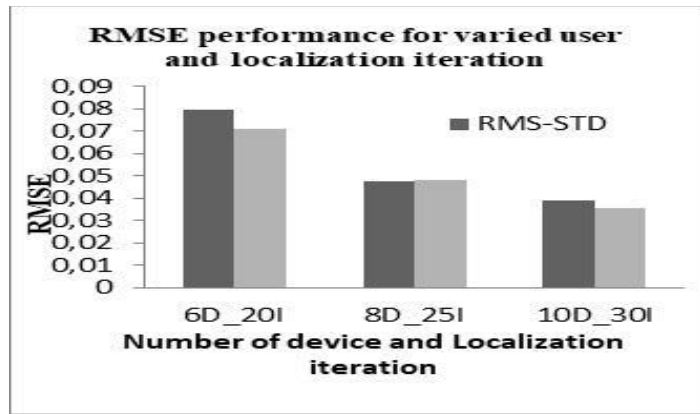

Figure 5. RMSE performance for varied devices and localization iteration

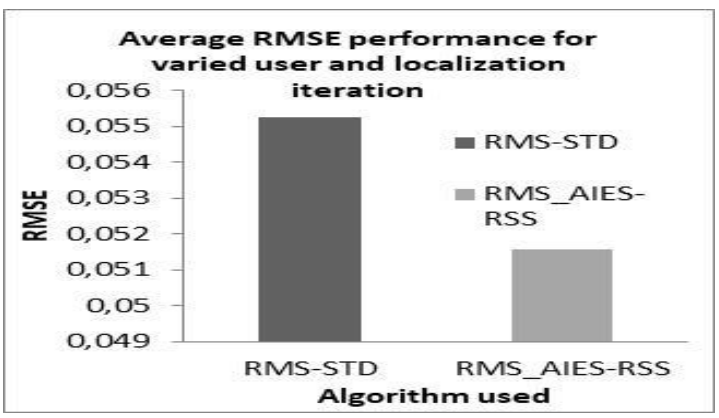

Figure 6. Average RMSE performance for varied number of sensor nodes and localization iteration

In our simulation study, to show the robust performance of the proposed localization algorithm, we have considered tolerance parameter for optimization. Performance is given in the Table 6 and the graphical representation of this is given in Figure 7. In this scenario of tolerance optimization we compared the standard algorithm for sensor localization with our proposed algorithm, by varying the tolerance optimization parameter. Proposed algorithm uses the tolerance parameter and achieves the optimal RMSE which is minimal when compared to the existing RSME standard algorithm. The average performance of the localization is given in Figure 8 and achieved simulated data is tabulated in Table 7.

Table 5. Tolerance Optimization Performance

\begin{tabular}{cc}
\hline Algorithm Used & Average RMSE \\
\hline RMS-STD & 0.055266667 \\
RMS-AIES-RSS (proposed) & 0.051566667 \\
\hline
\end{tabular}

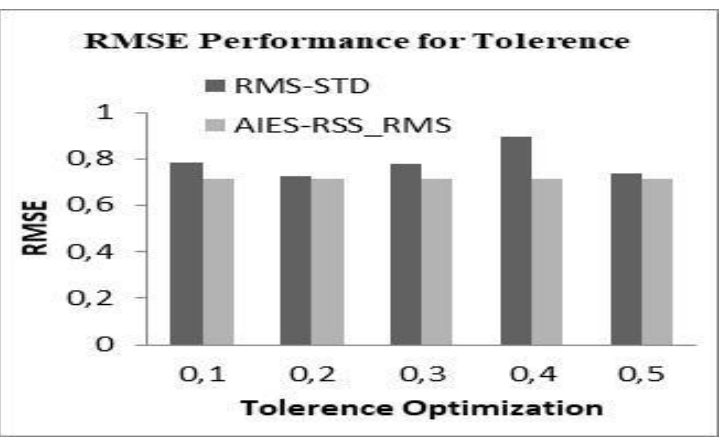

Figure 7. Tolerance optimization performance
Table 6. Average RMSE Performance

\begin{tabular}{ccc}
\hline $\begin{array}{c}\text { Tolerance } \\
\text { Optimization }\end{array}$ & RMS-STD & $\begin{array}{c}\text { AIES- } \\
\text { RSS_RMS }\end{array}$ \\
\hline 0.1 & 0.782 & 0.712 \\
0.2 & 0.723 & 0.712 \\
0.3 & 0.776 & 0.712 \\
0.4 & 0.895 & 0.712 \\
0.5 & 0.735 & 0.712 \\
Tolerance & RMS-STD & AIES- \\
Optimization & RSS_RMS \\
\hline
\end{tabular}

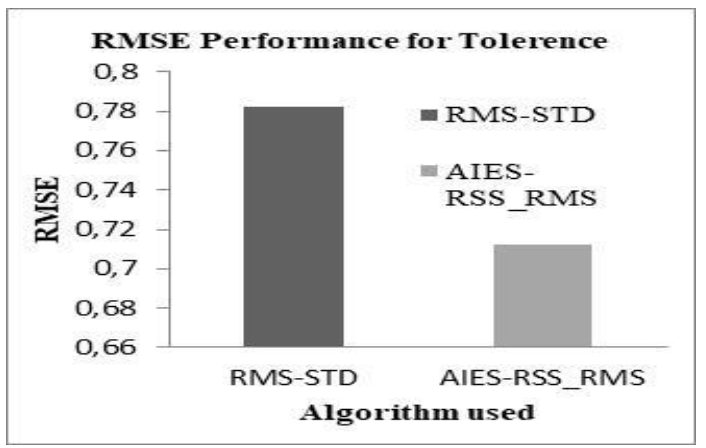

Figure 8. Tolerance optimization performance 


\section{CONCLUSION}

Wireless sensor network is a network that enables correspondence between various devices associated through a infrastructure protocol. Finding the position or location of sensor node (Localization) is an important factor in sensor network for providing efficient service to end user. The existing localization is not cost effective and efficient and it suffers from localization optimization overhead. The existing technique proposed so for suffers in estimating the likelihood of localization error. To cater this in this work the author proposes a RSS (Received signal strength) based localization technique and als o proposes an adaptive information estimation to reduce or approximate the localization error in wireless sensor network. The simulation results show the effectiveness of our proposed localization model over existing protocol in term of RMSE.

\section{REFERENCES}

[1] L. Xu, and X. Wang, "Compressed sensing signal and data acquisition in wireless sensor networks and Internet of Things," IEEE Trans. Ind. Informat., 2013, vol. 9, no. 4, pp. 2177-2186

[2] D. Guinard, V. Trifa, S. Karnouskos, P. Spiess, and D. Savio, "Interacting with the SOA-based Internet of Things: Discovery, query, selection, and on-demand provisioning ofWeb services," IEEE Trans. Services Comput., 2010, vol. 3, no. 3, pp. 223-235.

[3] D. Chiang, C. Lin, and M. Chen, "The adaptive approach for storage assignment by mining data of warehouse management system for distribution centres," Enterprise Inf. Syst., 2011 vol. 5, no. 2, pp. 219-234.

[4] L. Duan and L. D. Xu, "Business intelligence for enterprise systems: A survey," IEEE Trans. Ind. Informat., 2012, vol. 8, no. 3, pp. 679-687.

[5] L. D. Xu,W. Viriyasitavat, P. Ruchikachorn, and A. Martin, "Using propositional logic for requirements verification of service workflow," IEEE Trans. Ind. Informat., , 2012, vol. 8, no. 3, pp. 639-646.

[6] E. Xu, M. Wermus, and D. Bauman, "Development of an integrated medical supply chain information system," Enterprise Inf. Syst., 2011, vol. 5, no. 3, pp. 385-399.

[7] L. Duan, W. Street, and E. Xu, "Healthcare information systems: Data mining methods in the creation of a clinical recommender system," Enterprise Inf. Syst., 2011, vol. 5, no. 2, pp. 169-181.

[8] P. Biswas, K.-C. Toh, and Y. Ye, "A distributed SDP approach for largescale noisy anchor-free graph realization with applications to molecular conformation," SIAM J. Sci. Comput., Mar. 2008, vol. 30, no. 3, pp. 1251-1277.

[9] A. Boukerche, H. A. B. F. Oliveira, E. F. Nakamura, and A. A. F. Loureiro, "Localization systems for wireless sensor networks," IEEE Wireless Commun., 2007, vol. 14, no. 6, pp. 6-12,.

[10] L. D. Xu, "Enterprise systems: State-of-the-art and future trends," IEEE Trans. Ind. Informat., 2011, vol. 7, no. 4, pp. 630-640.

[11] X. Wang and S. Li, "Scalable routing modeling for wireless ad hoc networks by using polychromatic sets," IEEE Syst. J., 2013, vol. 7, no. 1, pp. 50-58.

[12] O.-H. Kwon and H.-J. Song, “Localization through map stitching in wireless sensor networks, " IEEE Trans. Parallel Distrib. Syst., Jan. 2008, vol. 19, no. 1, pp. 93-105.

[13] M. Hamdi, N. Boudriga, and M. S. Obaidat, "Bandwidth-effective design of a satellite-based hybrid wireless sensor network for mobile target detection and tracking,” IEEE Syst. J.,2008, vol. 2, no. 1, pp. 74-82.

[14] S. Lee, E. Kim, C. Kim, and K. Kim, "Localization with a mobile beacon based on geometric constraints in wireless sensor networks," IEEE Trans. Wireless Commun., 2009, vol. 8, no. 12, pp. 5801-5805.

[15] S. Misra, S. K. Dhurandher, M. S. Obaidat, N. Nangia, N. Bhardway, P. Goyal, and S. Aggarwal, "Node stabilitybased location updating in mobile Ad-Hoc networks,” IEEE Syst. J., 2008, vol. 2, no. 2, pp. 237-247.

[16] T. S. Rappaport, “Wireless Communications: Principles and Practice”. Englewood Cliffs, NJ: Prentice-Hall, 1996.

[17] Weile Zhang; Qinye Yin; Hongy ang Chen; Feifei Gao; Ansari, N., "Distributed Angle Estimation for Localization in Wireless Sensor Networks," in Wireless Communications, IEEE Transactions on , 2013, vol.12, no.2, pp.527-537.

[18] Malajner, M.; Gleich, D.; Planinsic, P., "Angle of Arrival Measurement Using Multiple Static Monopole Antennas," in Sensors Journal, IEEE, 2015, vol.15, no.6, pp.3328-3337.

[19] Quanrui Wei; Dexing Zhong; Jiuqiang Han, "Improved localization method based on multi-hop distance unbiased estimation," in Communications, IET , 2014, vol.8, no.16, pp.2797-2804.

[20] [20] Maddumabandara, A.; Leung, H.; Minxiang Liu, "Experimental Evaluation of Indoor Localization Using Wireless Sensor Networks," in Sensors Journal, IEEE , Sept. 2015, vol.15, no.9, pp.5228-5237.

[21] Ian F. Akyildiz, Weilian Su, Yogesh Sankarasubramaniam, and Erdal Cayirci,"A Survey on Sensor Networks"-Aug 2002, IEEE Communications Magazine.

[22] Abbas, A.M., "TrustLP: A trust-based localization protocol for wireless sensor networks," in Modeling and Optimization in Mobile, Ad Hoc, and Wireless Networks (WiOpt), 2015 13th International Symposium on , 2015, vol., no., pp.529-536.

[23] Z. Yang, Y. Liu, “Quality of Trilateration: Confidence-Based Iterative Localization”, IEEE Transactions on Parallel and Distributed Systems, 2010, vol. 21, no. 5, pp. 631-640.

[24] Lijun Liu, Yuankun Wei, Guoyi, “An Adaptive Hybrid Localization Algorithm for Wireless Sensor Networks", IEEE International conference on Instrumentation \& Measurement, Computer, Communication and Control, 2012, pp.1579-1582. 
[25] Qingguo Zhang, Jinghua Wang, “Localization Algorithm for Wireless Sensor Network Based on Genetic Simulated Annealing Algorithm”, IEEE international conference, 2008.

[26] Yuan Zhang, Yuehui Chen, Yue Liu, "Towards Unique and Anchor-Free Localization for Wireless Sensor Networks", Journal of wireless personal communications, 2012, VOL. 63, pp. 261-278.

[27] Anil Kumar, Arun Khosla, Jasbir Singh, Satvir Singh, "Meta-Heuristic Range Based Node Localization Algorithm for Wireless Sensor Networks", IEEE international conference, 2012.

[28] [Stephan H Chagas, Joao B. Martin, Leonardo L. de Oliveira, "An Approach to Localization Scheme of Wireless Sensor Networks Based on Artificial Neural Networks and Genetic Algorithm", IEEE International conference on new circuits and systems, 2012, pp. 137-140.

[29] RaghavaSrinivasaNallanthighal ,VeeranjaneyuluChinta, "Improved Grid-Scan Localization Algorithm for Wireless Sensor Networks",Hindawi Publishing Corporation Journal of EngineeringVolume 2014, Article ID 628161, 5 pages.

[30] Nicolescu, D. and Nath, B. (2001) Ad-Hoc positioning systems (APS). Proceedings of the 2001 IEEE Global Telecommunications Conference, San Antonio, 25-29 November 2001, 2926-2931. http://dx.doi.org/10.1109/GLOCOM.2001.965964

[31] Dai, Y., Wang, J.P. and Zhang, C.W. (2010) Research and Improvement of Localization Algorithms for Wireless Sensor Network. Journal of Transduction Technology, 23, 567-570.

[32] Benkic, K., Malajner, M., Planinsic, P. and Cucej, Z.. Using RSSI value for distance estimation in wireless sensor networks based on ZigBee, Proceedings of the 15th International Conference on Systems, Signals and Image Processing, IWSSIP 2008, Bratislava, Slovakia, 2008, pp. 303-306.

[33] Akyildiz, I. and Vuran, M. Wireless Sensor Networks, John Wiley \& Sons, West Sussex, 2010.

[34] Barsocchi, P., Lenzi, S., Chessa, S. and Giuntaa, G. Virtual calibration for RSSI-based indoor localization with ieee 802.15.4, Proceedings of the IEEE International Conference on Communications (ICC), Dresden, Germany, pp. 1-5, 2009.

[35] Beutel, J. (2005). Handbook of Sensor Networks Compact Wireless and Wired Sensing Systems, CRC Press, Boca Raton, FL, 2005.

[36] Karl, H. and Willig, A. Protocols and Architectures for Wireless Sensor Networks, John Wiley \& Sons, West Sussex, 2005.

[37] Motter, P., Allgayer, R., Müller, I. and de Freitas, E. Practical issues in wireless sensor network localization systems using received signal strength indication, Proceedings of the Sensors Applications Symposium (SAS), San Antonio, TX, USA, pp. 227-232, 2011.

[38] Liu, L. and E, M. (2010). Localization for wireless sensor networks by combining TFDA and FMCW, Proceedings of the IEEE International Conference on Mechatronics and Automation, Xian, China, 2010 pp. 945-950.

[39] Magnani, A. and Leung, K. Self-organized, scalable GPS-free localization of wireless sensors, IEEE WCNC, Hong Kong, China, 2007pp. 3798-3803.

[40] Zhang Xiaolong, Xie Hui-ying, Zhao Xiaojian wireless sensor networks in an improved DV-Hop localization algorithm. Journal of Computer Applications, 2007,pp 2672 -267,.

[41] Hongyang Chen; Sezaki, K.; Ping Deng; Hing Cheung So, "An improved DV-Hop localization algorithm for wireless sensor networks," in Industrial Electronics and Applications, ICIEA, 3rd IEEE Conference on , 2008, vol., no., pp.1557-1561,

[42] Hui-Min Cui; Ya-Fang Wang; Jin-Xing Liu; Li-Na Liu, "Research and improvement of DV_HOP localization algorithm in wireless sensor networks," in Machine Learning and Cybernetics (ICMLC), International Conference on, 2015.vol.2, no., pp.708-713.

[43] Xu Chun-Xia; Chen Ji-Yu, "Research on the improved DV_HOP localization algorithm in WSN," in Internation Journal of Smart Home (IJSH), 2015, vol.9, no.4, pp.157-162.

[44] Chandirasekaran D*, T. Jayabarathi," Wireless Sensor Networks Node Localization-A Performance Comparison of Shuffled Frog Leaping and Firefly Algorithm in LabVIEW", TELKOMNIKA Indonesian Journal of Electrical Engineering Vol. 14, No. 3, June 2015, pp. $516 \sim 524$

[45] Songhao Jia*, Cai Yang;" Received Signal Strength Indicator Node Localization Algorithm Based on Constraint Particle Swarm Optimization”, TELKOMNIKA (Telecommunication, Computing, Electronics and Control), Vol.13, No.1, March 2015, pp. 221 229 